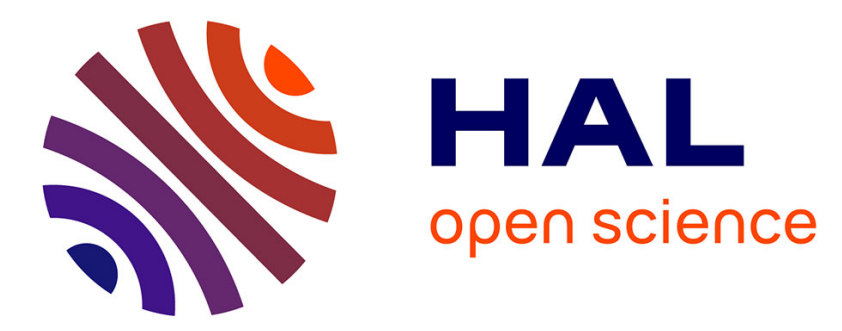

\title{
Highly anisotropic epitaxial L10 FePt on Pt(001)
}

Marcio Soares, Hélio C. N. Tolentino, Maurizio de Santis, Aline Y. Ramos, Julio C. Cezar

\section{To cite this version:}

Marcio Soares, Hélio C. N. Tolentino, Maurizio de Santis, Aline Y. Ramos, Julio C. Cezar. Highly anisotropic epitaxial L10 FePt on Pt(001). Journal of Applied Physics, 2011, 109, pp.07D725. 10.1063/1.3556782 . hal-00631591

\section{HAL Id: hal-00631591 \\ https://hal.science/hal-00631591}

Submitted on 12 Oct 2011

HAL is a multi-disciplinary open access archive for the deposit and dissemination of scientific research documents, whether they are published or not. The documents may come from teaching and research institutions in France or abroad, or from public or private research centers.
L'archive ouverte pluridisciplinaire HAL, est destinée au dépôt et à la diffusion de documents scientifiques de niveau recherche, publiés ou non, émanant des établissements d'enseignement et de recherche français ou étrangers, des laboratoires publics ou privés. 


\title{
Highly anisotropic epitaxial $L 1_{0} \mathrm{FePt}$ on $\mathrm{Pt}(001)$
}

\author{
Márcio M. Soares, ${ }^{1, a)}$ Hélio C. N. Tolentino, ${ }^{1, b)}$ Maurizio De Santis, ${ }^{1}$ Aline Y. Ramos, ${ }^{1}$ \\ and Júlio C. Cezar ${ }^{2}$ \\ ${ }^{1}$ Institut Néel, UPR 2940_CNRS, 25 av. des Martyrs, BP166, 38042 Grenoble, France \\ ${ }^{2}$ European Synchrotron Radiation Facility, 6 rue Jules Horowitz, BP220, 38043, Grenoble, France
}

(Presented 17 November 2010; received 6 October 2010; accepted 24 November 2010; published online 1 April 2011)

We report on the structure and magnetism of an ultrathin $L 1_{0}$ FePt film epitaxially grown on $\mathrm{Pt}(001)$ single crystal. This film presents an enhanced tetragonal distortion and a quite large magnetic anisotropy, with an orbital contribution to the Fe total magnetic moment as large as $10 \%$. (c) 2011 American Institute of Physics. [doi:10.1063/1.3556782]

\section{INTRODUCTION}

FePt alloy in the $L 1_{0}$ chemically ordered phase has attracted much attention as a candidate for ultrahigh density magnetic storage media. Beyond the influence of chemical order on the magnetic properties of $\mathrm{FePt}$, it has been recently proposed $^{1}$ that variation in tetragonal $c / a$ ratio can induce new magnetic phases to this alloy. $\mathrm{FePt}$ and $\mathrm{FePt} / \mathrm{MnPt}$ thin films were grown on a $\mathrm{Pt}(001)$ single crystal and in situ studied by grazing incidence $\mathrm{x}$-ray diffraction (GIXRD) at the ultra-high vacuum station of the French CRG BM32 beamline at European Synchrotron Radiation Facility (ESRF), Grenoble, France. The station is equipped with standard tools for surface preparation, film deposition, and characterization. Prior to metal deposition, the $\operatorname{Pt}(001)$ surface was cleaned by several cycles of Ar sputtering and annealing (at $1173 \mathrm{~K}$ for $5-10 \mathrm{~min}$ ), followed by annealing under oxygen $\left(1.2 \times 10^{-7} \mathrm{mbar}_{2}\right.$ at $973 \mathrm{~K}$ for $\left.5 \mathrm{~min}\right)$ then flash-annealing at $1173 \mathrm{~K}$ before slowly cooling down. The cleanness of the surface was checked by Auger spectrometry and by the appearance of the well-known $\mathrm{Pt}(001)$-hex reconstruction. ${ }^{2}$ $\mathrm{X}$-ray diffraction was measured with $20 \mathrm{keV}$ monochromatized photons under a grazing incidence angle of $0.6^{\circ}$, about three times the critical angle for total reflection. Clean $\mathrm{Pt}(001)$ reconstructs to form a closed packed hexagonal monolayer on top of the bulk square planar symmetry. This monolayer is incommensurate in both directions within the surface plane and is about $25 \%$ denser than the bulk square surface. Rocking scans about the Pt(001)-hex peaks confirm the presence of the two equivalent domains of the reconstruction. From in-plane transverse scans the reconstruction coherent lengths have been estimated to be around $20 \mathrm{~nm}$, while the crystal has shown large terraces on the order of $300 \mathrm{~nm}$. High purity Fe and Pt were deposited from watercooled electron beam evaporators, the pressure rising up to $5 \times 10^{-10} \mathrm{mbar}$ while operating the sources. The evaporation rate was calibrated with a quartz crystal micro balance, and cross-checked measuring the oscillation period of the antiphase $\mathrm{x}$-ray reflectivity intensity during a calibration deposi-

\footnotetext{
${ }^{a)}$ Electronic mail: marcio.soares@grenoble.cnrs.fr.

${ }^{\mathrm{b})}$ Electronic mail: helio.tolentino@grenoble.cnrs.fr.
}

tion. A deposition rate of 0.30 and $0.04 \mathrm{ML} / \mathrm{min}$ was established for $\mathrm{Fe}$ and $\mathrm{Pt}$, respectively.

Local structure of the layers was probed by $\mathrm{x}$-ray absorption spectroscopy (XAS) at the French CRG BM30B FAME beamline at ESRF. XAS spectra were collected in the fluorescence mode using a 30-element Canberra Ge solid state detector, in the XANES (x-ray absorption near edge structure) and EXAFS (extended x-ray absorption fine structure) range. The $\mathrm{X}$-ray natural linear dichroism of the sample was investigated by collecting data with the electric field aligned in-plane (IP) and out-of-plane (OP) to the film's surface. Polar magneto-optical Kerr effect (MOKE) measured at room temperature (RT) confirms the out-of-plane anisotropy (inset in Fig. 1). X-ray magnetic circular dichroism (XMCD) at the $\mathrm{Fe} \mathrm{L}_{2,3}$ edges was performed at the ID08 beamline at ESRF.

A 2.0-nm FePt layer was prepared by alternate $\mathrm{Fe}$ and $\mathrm{Pt}$ thermal deposition. The $\operatorname{Pt}(001)$-hex substrate was kept at $573 \mathrm{~K}$ during the sample preparation to favor $L 1_{0}$ chemical order with $\mathrm{c}$-axis perpendicular to the surface. ${ }^{3}$ Alternate deposition of $\mathrm{Fe}$ and Pt comes to favor ordering. ${ }^{3,4}$ Recently, it was reported that during $\mathrm{Fe}$ deposition on $\mathrm{Pt}(001)$-hex at $\mathrm{RT}$, an atomic site exchange process takes place between $\mathrm{Fe}$ (higher surface energy) and Pt substrate atoms. ${ }^{5}$ Up to 1.2 MLs of Fe deposition, no evidences of Fe atoms on the surface or Fe subsurface islands were found. In the case of deposition at $573 \mathrm{~K}$ this atomic exchange process is expected to be reinforced. Accordingly, a sample with an excess in Fe, consisting of $[\mathrm{Fe}(2 \mathrm{ML}) / \mathrm{Pt}(1 \mathrm{ML})]_{3}$, was deposited. After cooling down to RT, sets of nonspecular crystal truncation rods (CTR) were measured. Figure 1 shows the x-ray scattering along the $\left(\begin{array}{lll}1 & 1 & l\end{array}\right)$ CTR $(l$ is the out-of-plane momentum transfer in reciprocal lattice units of the Pt substrate). The oscillations are preserved up to high $l$ values. Moreover, no evidence of in-plane relaxation was observed by $(h, k)$-scans in the reciprocal space. Both outcomes confirm a pseudomorphic growth of the layer. The FePt layer adopts the in-plane lattice parameter of $\mathrm{Pt}$ substrate, $a_{\mathrm{Pt}}=3.924 \AA$. The strong peak around $l=2.2$ (Fig. 1) arises from the out-of-plane chemically ordered $L 1_{0} \mathrm{FePt}$ layer. No trace of $L 1_{2}$ phase was found. The tetragonality of the epitaxial FePt layer is $c / a=0.907 \pm 0.002$, averaged from the whole set of CTRs. Such strong structural anisotropy has never been reported before for epitaxial $L 1_{0} \mathrm{FePt}$ thin films. One should note that 


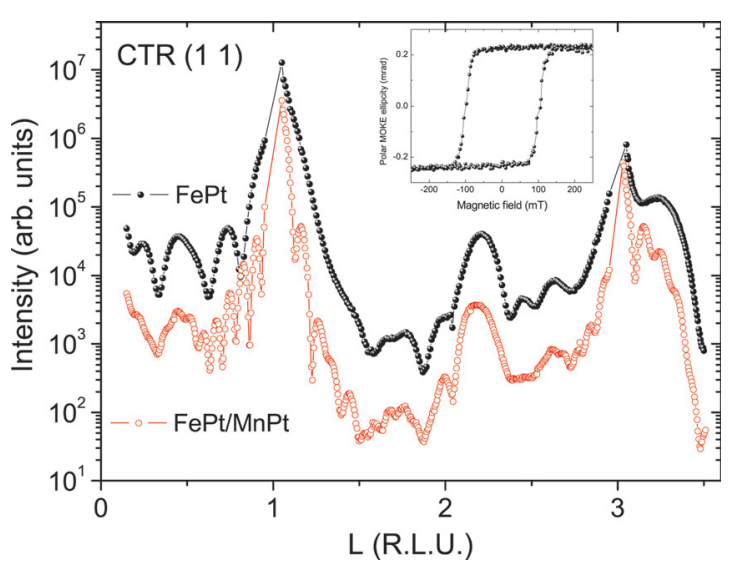

FIG. 1. (Color online) X-ray scattering along the $\left(\begin{array}{lll}1 & 1 & l\end{array}\right)$ CTR for the $\mathrm{FePt}(2.0 \mathrm{~nm})$ layer before (upper spheres) and after (bottom circles) being covered by $\operatorname{MnPt}(3 \mathrm{~nm})$. Inset: RT polar MOKE hysteresis loop showing $100 \mathrm{mT}$ coercitivity and $0.2 \mathrm{mrad}$ MOKE ellipticity at remanence.

FePt $L 1_{0}$ alloy has lattice parameters of $a=3.860 \AA$ and $c=3.713 \AA(c / a=0.96) .{ }^{6}$ The pseudomorphic FePt layer has a tensile strain of $1.7 \%$.

Quantitative analysis of the GIXRD data was done by simulating and fitting the experimental CTRs. The fitting parameters are the number of layers, the distances among them, the degree of chemical order and the Fe concentration. The best fitting model consists of $12 \mathrm{FePt}$ ML $(2.0 \mathrm{~nm})$ and is divided into two parts. The first part is an interdiffusion layer composed by four chemically disordered MLs and corresponds to scattered Fe atoms into the Pt matrix. The second part consists of 8 chemically ordered MLs of FePt. The fitting procedure did not allow a precise determination of the $\mathrm{Fe}$ concentration. Possible values range from 0.5 to 0.65 . However, the sample stoichiometry can be more precisely determined from the unit cell volume $V=54.802 \AA^{3}$. This value is in excellent agreement with that of ordered $\mathrm{Fe}_{55} \mathrm{Pt}_{45}$ nanocrystalline alloy ${ }^{6} V_{0.55}=54.807 \AA^{3}$. This stoichiometry was used as an input parameter in the model. The order parameter for the 8 ordered MLs is $S=0.74 \pm 0.05$. If one considers the whole film (4 ML Fe diffusion +8 ordered $\mathrm{FePt}$ ) the order parameter is reduced to $S=0.5 \pm 0.05$.

As a second step of sample preparation, the layer was covered by a 3.0-nm MnPt film. The (11) CTR is presented in Fig. 1. Finally, the sample was covered by a $1.2-\mathrm{nm} \mathrm{Pt}$ cap-layer for further ex situ studies.

Local anisotropy is clearly observed in XAS measurements at the Fe $\mathrm{K}$ edge. In the XANES spectra of metallic compounds, the double resonance (A and $\mathrm{B}$ ) just above the absorption edge is an experimental signature of the structures fcc or $L 1_{0}$ and small differences in the relative height of these peaks are associated to variations in the local chemical order. $^{7,8}$ The significant difference in these amplitudes for in-plane and out-of-plane XANES (Fig. 2) reveal the anisotropy in the local order. In addition the position of the first EXAFS oscillation (C), around $7145 \mathrm{eV}$, is slightly shifted toward higher energies for the IP spectra with respect to the $\mathrm{OP}$ one. This shift corresponds to a shortening of the distance to the first neighbors in the [001] direction. The analysis of the EXAFS signal (inset in Fig. 2) gives access to the local

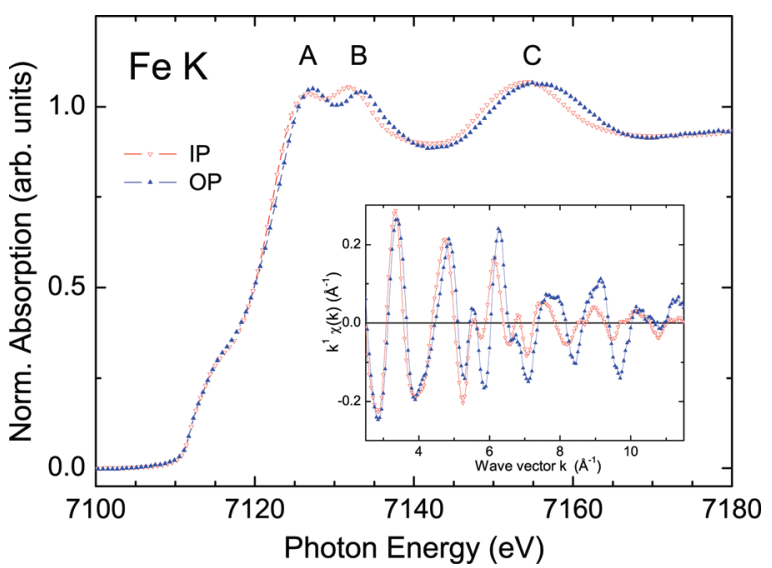

FIG. 2. (Color online) Polarization dependent XANES in the Fe K edge for $\mathrm{X}$-ray beam polarization along the surface plane (IP) and out-of-plane (OP). Inset: structural anisotropy seen in the EXAFS oscillations.

structural anisotropy. The distance to the first neighbors is $2.635 \pm 0.005 \AA$ in the film plane and $2.745 \pm 0.005 \AA$ along the [001] direction, giving a $c / a$ ratio value of $0.915 \pm 0.005$, in full agreement with the in situ GIXRD results.

The spin and orbital contributions to the total moment of $\mathrm{Fe}$ in the FePt layer were investigated by using element-selective XMCD spectroscopy. Figure 3 shows the normalized XAS and XMCD spectra at the $\mathrm{L}_{2,3}$ edges recorded at $\mathrm{RT}$ in total electron yield mode. The incident beam direction was normal to the sample surface. An applied magnetic field of 1 $\mathrm{T}$ ensures nearly $100 \%$ saturation of Fe moments. XMCD spectra are obtained by reversing the circularly polarized light. According to the sum rules, the effective spin $\left(m_{s}^{\text {eff }}\right)$ and orbital $\left(m_{l}\right)$ magnetic moments can be obtained by performing adequate integrals in the spectra. ${ }^{9}$ The ratio $m_{l} / m_{s}^{\text {eff }}$ is proportional to the deviation from zero of the integral of the XMCD signal (dashed line in Fig. 3). To obtain absolute values for $m_{l}$ and $m_{s}^{\text {eff }}$ the excitations to $3 d$ states must be separated from those to the continuum. A two-step function (dotted line in Fig. 3) is usually subtracted from the XAS spectrum. The broad feature at $736 \mathrm{eV}$ corresponds to a resonance of the $P t 4 s$ electron excitation to the continuum of states $\left(P t N_{1}\right.$

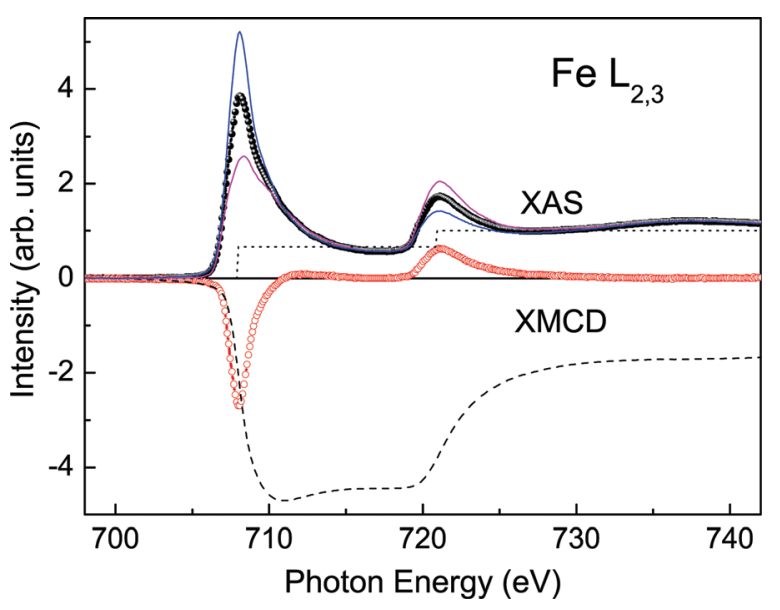

FIG. 3. (Color online) Normalized XAS (closed circles) and XMCD (open circles) spectra at the $\mathrm{Fe}_{2,3}$ edge of the sample. The integrated XMCD signal is shown by the dashed line. 
edge). This excitation does not contribute to the XMCD signal, but affects the normalization and, consequently, the absolute values for $m_{l}$ and $m_{s}^{\text {eff }}$. To compare with data in literature, ${ }^{10,11}$ we have subtracted the two-step function, taken the normalization energy around $730 \mathrm{eV}$ and integrated the $3 d$ states up to that energy. The additional contribution of the $\mathrm{Pt}$ N1 edge has also been simulated. The experimental data reduction (background and normalization) gives an estimated error bar around $15 \%$ on $m_{l}$ and $m_{s}^{e f f}$ and smaller than $10 \%$ for the ratio. Taking the theoretical number of $d$ holes, $n_{d}=3.705,{ }^{12}$ we have found $m_{l}=0.29(4) \mu_{B}$ and $m_{s}^{\text {eff }}=2.6(4) \mu_{B}$. The values given by band structural calculations are $m_{l}=0.072 \mu_{B}$ and $m_{s}^{\text {eff }}=2.87 \mu_{B}{ }^{12}$ Antoniak et al. ${ }^{10}$ found an enhanced orbital magnetism in 6-nm FePt nanoparticles when chemical order is improved by annealing $\left(m_{l} / m_{s}^{\text {eff }}=0.093, m_{l}=0.24 \mu_{B}\right.$, and $\left.m_{s}^{\text {eff }}=2.59 \mu_{B}\right)$. In the case of thin films, the largest orbital contribution is reported for films grown on a $\mathrm{MgO}(001)$ substrate at $350^{\circ} \mathrm{C}$ $\left(m_{l} / m_{s}^{\text {eff }}=0.099, m_{l}=0.24 \mu_{B}\right.$, and $\left.m_{s}^{\text {eff }}=2.45 \mu_{B}\right){ }^{11}$ These films presented the $L 1_{0}$ chemical order with lattice parameters of $a=3.870 \AA$ and $c=3.714 \AA(c / a=0.96)$, very close to the bulk values. Our pseudomorphic film $(c / a=0.91)$ shows an even larger orbital contribution, $m_{l} / m_{s}^{\text {eff }}=0.116 \pm 0.012$, which is the largest experimental orbital to spin moments ratio ever found in FePt ordered alloys.

We have shown that FePt films grow pseudomorphically on a $\mathrm{Pt}(001)$ substrate. We interpret the enhanced orbital magnetism observed in our film as a result of the increased tetragonal distortion and the high degree of chemical order.

The authors acknowledge the European Synchrotron Radiation Facility for beamtime at BM32, ID08, and BM30B beamlines. MMS is supported by a grant of Fondation Nanosciences.

${ }^{1}$ Z. Lu, R. V. Chepulskii, and W. H. Butler, Phys. Rev. B 81, 094437 (2010).

${ }^{2}$ D. Gibbs, G. Grübel, D. M. Zehner, D. L. Abernathy, and S. G. J. Mochrie, Phys. Rev. Lett. 67, 3117 (1991).

${ }^{3}$ T. Shima, T. Moriguchi, S. Mitani, and K. Takanashi, Appl. Phys. Lett. 80, 288 (2002).

${ }^{4}$ S. Imada, A. Yamasaki, S. Suga, T. Shima, and K. Takanashi, Appl. Phys. Lett. 90, 132507 (2007).

${ }^{5}$ K. He, L. J. Zhang, X. C. Ma, J. F. Jia, Q. K. Xue, and Z. Q. Qiu, Phys. Rev. B 72, 155432 (2005).

${ }^{6}$ J. Lyubina, I. Opahle, M. Richter, O. Gutfleisch, K.-H. Muller, L. Schultz, and O. Isnard, Appl. Phys. Lett. 89, 032506 (2006).

${ }^{7}$ N. M. Souza-Neto, A. Y. Ramos, H. C. N. Tolentino, A. Martins, and A. D. Santos, Appl. Phys. Lett. 89, 111910 (2006).

${ }^{8}$ A. Martins, N. Souza-Neto, M. Fantini, A. Santos, R. Prado, and A. Ramos, J. Appl. Phys. 100, 013905 (2006).

${ }^{9}$ C. T. Chen, Y. U. Idzerda, H.-J. Lin, N. V. Smith, G. Meigs, E. Chaban, G. H. Ho, E. Pellegrin, and F. Sette, Phys. Rev. Lett. 75, 152 (1995).

${ }^{10}$ C. Antoniak, J. Lindner, M. Spasova, D. Sudfeld, M. Acet, M. Farle, K. Fauth, U. Wiedwald, H.-G. Boyen, P. Ziemann, F. Wilhelm, A. Rogalev, and S. Sun, Phys. Rev. Lett. 97, 117201 (2006).

${ }^{11}$ C. J. Sun, G. M. Chow, G. H. Fecher, J. S. Chen, H.-J. Lin, and Y. Hwu, Jpn. J. Appl. Phys. 45, 2539 (2002).

${ }^{12}$ I. Galanakis, M. Alouani, and H. Dreyssé, Phys. Rev. B 62, 6475 (2000). 